\title{
Biosensors in immunology: the story so far
}

\author{
Sulabha S. Pathak and Huub F.J. Savelkoul
}

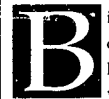

iosensors can be defined as devices that combine a biological recognition mechanism with a suitakls iranstucer, which gencrite: a masuralble signa! in response to changes in concentration of a given biomolecuir at the detector surface. Evanescent optical sensing devices, which rely upon the evanescent field to probe changes occurring at the sensor surface as a result of binding, are being increasingly used in immunology'. Several different types of evanescent field biosensors have become commercially available (Box 1 ). The most common sensor surface is a flexible hydrogel matrix composed of carboxymethylated dextran chains of a thickness of 100-200 nm. It is possible to inamobilize various molecules to this dextran layer by conventional chemical methods. The ligand may be one of the reactants in the interaction of interest, a capture antibody having a high affinity for one of the reactants, or even streptavidin to which the biotinylated ligand is later adsorbed. Recently, stir-coated polystyrene surfaces have become available. It is possible to coat such surfaces by simple adsorption. The polystyrene surfaces may have an advantage over the dextran surfaces in that they are flat, unidimensional surfaces. However, many of the criticisms directed against enzyme-linked immunosorbent assay (ELISA) surfaces (denaturation of proteins. changes in protein conformation, desorption, etc.) are also valid against such polystyrene surfaces. This article discusses the scope and limitations of four major areas of application of optical biosensors: concentration determination, kinetic interaction analysis, epitope mapping and DNA-ligand interaction analysis.

\section{Concentration determination}

Biosensors offer the possibility of quick concentration determination of the analyte. Concentration determinations in immunoassays are generally based on equilibrium

\author{
Optical biosensors an finding \\ inger of applications its \\ iminumology. They chable
}

biomolecular interactions to be

chancterized in real time without

the neted to labed reactants, and,

berause intizitual binding steps

can be alsualized, are particularly

silited to complex assays.

measurements of immune complexes. Solidphase immunoassays such as ELISAs or radioimmunoassays (RIAs) require the labeling of one of the reactants, are timeconsuming, discontinuous techniques and comprise cycles of washes during which the primary antibody/antigen might dissociate. They are therefore influenced by the affinity of the reaction ${ }^{2}$. Moreover, adsorption to the plastic surface is found to denature many proteins or disrupt their quaternary structure ${ }^{3}$. Also, mass transport iimitations may lead to under-estimation of the analytet.

In biosensors, the response can be measured either directly as that of a ligate exposed to the biospecific surface, or secondarily as the response from a subsequently introduced ligate-specific reagent. Tise use of the secondary reagent enhances the signal. It also increases the specificity of the system. Biosensors suffer from one major constraint also shared by solid-phase immunoassays: a high percentage of immobilized molecules may not be in the correct conformation for interaction. The use of the biotin-streptavidin system can help in overcoming this constraint by increasing the sensitivity and the specificity of the assay.

Biosensors are especially useful where concentration determination is desired in small volumes and hands-on time is limited. Moreover, biosensors can measure biomolecules quantitatively at a single dilution. Furthermore, the sensor surface can be regenerated, so that multiple determinations can be done on the same surface. Adjusting the flow rate or the stirrer rate helps reduce the mass transport limitations observed in E:.LSAs, and such adjustments are also useful in biosensors. However, other parameters such as diffusion of the analyte in the dextran matrix and aftinity of the secondary reagents may influence determinations in the biosensor, although the latter constraint can be minimized by the use of high-affinity secondary reagents. Shuck ${ }^{5}$ has suggested that using a considerably thinner dextran matrix could minimize the mass transport limitations in the biosensor, because the size of the gel is the single most important variable for the mass transport within the gel. The use of aminosilane surfaces or direct linking of the biomolecule of interest to the sensor chip may help overcome some mass transport limitations, but it is difficult to immobilize significant amounts of proteins to such surfaces ${ }^{6}$.

Most of the published studies of the use of biosensors for concentration determination or interaction analysis use purified or semi-purified samples. When the protein of interest is in very small concentrations as compared with the other bulk proteins, mass transfer limitations could seriously hamper concentration determinations. The published reports generally indicate a detection limit in the nanogram to microgram range. This detection limit may not be low enough to permit analysis of molecules that are normally present in low concentration, such as rare antibody types, cytokines and cytokine receptors. Furthermore, since the biosensors read the changes in the sensor surface (refractive index or resonance) that occur as a result of binding/dissociation of biomolecules, the current instruments are not suitable for determining the interaction between very small molecules (molecular mass in the range of $5 \mathrm{kDa}$ ) directly. Biosensurs have been successfuily used to monitor Fv secretion in an Escherichia coli fermentation ${ }^{7}$ and for the determination of the concentration of antibodies against human inmunodeficiency virus 1 in seium". As judged from the pubiished reports, the anost popular application of the instrument 
seems to be kinetic interaction analysis rather than concentration determination.

\section{Kinetic interaction analysis}

Antigen-antibody interactions have traditionally been measured using a variety of methous such as RIA, ELISA and fluorescence quenching ${ }^{4}$. All these approaches have many theoretical and practical drawbacks ${ }^{4,10}$. When information regarding the kinetics of the reaction per sce is required, rapid kinetic measurements are desired and the traditional methods may not be suitable "1". Biosensors have been successfully used for such measurements'. A detailed theoretical consideration of kinetic analysis and especially the mathematics involved is given in Refs 11 and 12.

One of the major problems of kinetic interaction analysis by optical biosensors is the biphasic nature of both the association and the dissociation phases, which has been thought to be intrinsic to dextran surfaces ${ }^{11.13}$. However, such biphasic dissociation may not be restricted to dextran sur faces, since Mattes ${ }^{14}$ reports observing biphasic curves for antibodies dissociating from cell surfaces, with one fraction that dissociates rapidly and the otker at a much lower rate. Thus, a defizitive explanation for this effect cantut be provided at this point of time, and may be attributed to the bivalent nature of the antibody.

Box 2 lists some of the factors likely to cause deviations from Langmuirian approximations of the biosensor data. These numerous factors may be operative in different degrees depending on the instrument used as well as the characteristics of the interaction under investigation. However, with an understanding of the possible artefacts that can interfere with biosensor measurements, it is possible to obtain relevant kinetic information for interactions between a variety of biomolecules such as antigens and antibodies', adhesion molecules ${ }^{15}$ and cytokines/hormones and their receptors ${ }^{16.17}$. Association rate constants in the range $10^{3}-10^{6} \mathrm{M}^{-1} \mathrm{~s}^{-1}$ and dissociation rates of $10^{-5}-10^{-2} \mathrm{~s}^{-1}$ are within the instrument's capability's. Determining equilibrium constants of molecules with high dissociation rates is especially difficult with conventional

\section{Box I. Essential features of commercial evanescent field biosensors}

Surface plasmon resonance (SPR) biosensors

Examples: BIAcore and BIAlite (Pharmacia Biosensor AB, Uppsala, Sweden); IBIS

(Intersens Instruments B.V., Amersfoort, The Netherlands)

- Sensors consist of a thin metal layer (about $50 \mathrm{~nm}$ ) deposited on glass

- Evanescent wave generated by total internal reflection interacts with free oscillating electrons (called plasmons) in the metal film surface, resulting in resonance

- Light energy is lost to the film and the reflected light intensity drops sharply when resonance occurs

- Conditions for exciting resonance are extremely sensitive to any changes in the sensing layer

- Reactions occurring at the sensing layer can be followed by monitoring the resonance position

- The response from the instrunent is measured in terms of resonance signal

\section{Resonant mirror (RM) biosensors}

Example: LAsys (Fisons Applied Sensing Technoiogy, Cambridge, UK)

- Waveguiding technique devices

- Sensing layer is placed in the evanescent region of a dielectric waveguide structure

- A dielectric resonant layer of high refractive index that is separated from the prism by a layer of low refractive index is present at the sensing surface

- At the resonant point, light penetrates into the high index layer and propagates some distance along the sensing interface before coupling back in the prism

- Angle of excitation of resonance is very sensitive to changes at the sensing interface

- Change in the angle of resonance (in terms of arc.seconds) can be directly related to the presence and concentration of biomolecules

techniques'. This kind of kinetic analysis is particularly useful in the development of recombinant antibody technology and iisa been achieved using biosensors in, for example, the development of high-affinity antibudies fron a nave library by chain shuffling ${ }^{14}$. Biosensors have also been used in the development of phage libraries, where it is essential to determine the affinity of the displayed $\mathrm{Fv}$ fragments and their homologous antigens ${ }^{211}$.

Most of the published reports of kinetic interaction analysis between antigens and antibodies are restricted to monoclonal preparations. Indeed, reliable determination of $K_{u m}$ seems more difficult for polyclonal sera than for pure monoclonal antibody preparations of similar concentrations because of the heterogeneous affinities present in the former (S.S. Pathak and H.F.J. Savelkoul, unpublished). Determination of $K_{\text {dim }}$ is also problematic for polyclonal sera, because of failure to achieve appreciable dissociation (S.S. Pathak and H.F.J. Savelkuul, unpublished). Probably, high-affinity antibodies in the serum reassociate with the immobilized antigen because the diffusion of the unbound ligate away from the surface is rate limiting. This problem of reassociation could be less obvious in biosensors where a constant flow of liquid is maintained over the sensor surface. However, reassociation that can occur within the dextran matrix cannot be eliminated.

O'Shannessey and Winzor" are of the opinion that many factors could be responsible for deviations from pseudo-first-order kinetics in the biosensor and are perhaps operative to different degrees depending on the characteristics of the interaction anter investigation. They therefore suggest ,hat all the rate constants cobtained in biosensors are apparent and phenomenological. Various methods of data treatment are suggested in the literature to obtain reliable affinity constants ${ }^{11,12}$. However, such treatments are complicated and may not be able to account for all the factors involved. It is therefore 


\section{Box 2. Factors that can influence kinetic data obtained from the biosensor}

\section{inass transfer effects}

Result from:

- Bulk transport (i.e. transport to the sensor surface); can be minimized by adjusting flow rates

- Transport within the dextran matrix; difficult to detect and eliminate

\section{Ligand site heterogeneity}

- Arises from random covalent coupling; some immobilized ligand molecules may not be in the correct conformation

- Ligand sites deep within the gel are not easily accessible and therefore have an apparent lower affinity than the accessible sites

\section{Parking problems}

- Potential binding sites within the dextran matrix may be masked when a large ligate molecule binds to immobilized ligand; can be reduced with lower densities of immobilization

\section{Complex interactions}

- Bivalency/multivalency of either the ligate or the ligand leading to deviation from $1: 1$ stoichiometry

- Cooperativity (positive or negative) between the ligate/ligand

- Rebinding of the dissociated ligate molecules

suggested that the term $k_{\text {app }}$ or $k_{\text {abs }}$ be used to describe the kinetic events rather than describing them in terms of individual kinetic constant $\mathrm{s}^{21}$. With proper precautions and data handling, the observed affinity constants, however, are thought to be a direct reflection of the actual $K_{\mathrm{d}}$ and are in agreement with the data obtained from other methods of interaction analysis $s^{\text {. }}$. Thus, evanescent field biosensors can be easily used where determination of affinity constants is desired for relatively pure samples not containing a mixture of ligate molecules, and especially where various preparations are to be compared

Using biosensor technology, it is possible to study association and dissociation rates separately. This is especially useful in studying interactions of cell-surface receptors and their ligands. This tecluwology is therefore eminently suitable for studying the interaction of cytokines, growth hormones and other factors with their receptors. Until the introduction of biosensors, such studies were limited to conventional cellular bioassays vith thic associated problems of specificity, variability, longer duration and so on. With multi-unit receptors, it is possible to immobilize the receptor subunit ectodomains to the sensor surface (either individually or in combination) and perform kititite izter action analysis to arrive at the binding constants ${ }^{22.23}$ - studies that are virtually impossible with conventional techniques. Biosensors have also been used to study models of chain recognition and orientation of cytokines while they are reacting with their receptors ${ }^{24}$. Mutants containing specific residues can also be rapidly tested, allowing identification of regions on the molecules that influence the affinity of the interaction ${ }^{23,24}$. Recently, Stampfli et al. ${ }^{25}$ have even extended the use of this technology to study antigen-specific inhibition of IgE binding to the high affinity FceRI receptor, while Shen et al..$^{2 h}$ used the technology for the determination of local and global determinants for the interaction of interleukin 4 with its receptor. The cuvette design of the IAsys (Box 1) could, in theory, allow the immobilization of whole cells to the sensor surface and therefore the study of proteinc, lil interactions. However, the detection limit of the available instruments is not low enough to allow such measurements at present.

\section{Epitope mapping}

Biosensors are especially useful in epitope mapping, since it is possible to perform multisite analysis in which many relationships can be established in a single run by sequential addition of antibodies or other binding molecules. The open structure of the dextran matrix makes the analysis of the formation of large functional complexes feasible. Since it is possible to immobilize molecules so that they retain biological reactivity and conformation after regenerating the sensing layer, the process can be repeated using a different sequence of injections until all possible combinations are examined. Above all, the system allows the visualization of each successive binding step".

In their studies on the epitope-mapping of granulocyte-macrophage colony-stimulating factor, Laricchia-Robbio et al. ${ }^{27}$ have compared the applicability both of ELISA and biosensor technology to epitope mapping, to the clear advantage of the latter. Levy of al ${ }^{2 R}$ have used the technique to establish the major immunodominant epitope on the Goodpasture antigen as well as the heterogenetty of the autoantibody response in Goodpasture's disease. Similarly, Stemmer et $a .^{29}$ have used the technique to study the crossreactivity of anti-nucleosome autoantibodies for double-stranded DNA and histone $\mathrm{H3}$

\section{DNA-ligand interaction analysis} Understanding DNA-DNA and DNA-RNA interactions, as well as those between DNA and DNA-binding proteins, is becoming increasingly important in elucidation of immunological phenomena. Mobility or band-shift assays have been commonly used to study the interaction between DNA and DNA-binding proteins. However, these are discontinuous, time-consuming assays that are hampered by the need for recording a rapid reaction with a small number of data points and cannot provide a real-time picture of the associa'.ion events ${ }^{\text {,hl }}$. Biosensors have multiple advantages in this respect. The rate of ligand binding and the sequence 
specificity can be assayed easily and with high precision in a biosensor. Since the interactions can be monitored in real time, it is possible to optimize key steps during the procedure, which is not possible by other solid-phase gene assembly methods ${ }^{21}$. However, it is difficult to assay DNA-binding proteins in crude cell extracts by the currently available biosensors. Biosensors also require more protein for the binding analysis as compared with other methods ${ }^{3 n}$.

The most common method of immobilizing small oligomers of DNA is by introducing a molecule of biotin at the $3^{\prime}$ or the $5^{\prime}$ end and using a streptavidin-dextran surface as an efficient and robust capturing agent. The sensor can then be employed to study the kinetics of hybridization ${ }^{31}$, elongation by reverse transcriptase ${ }^{22}$, and multistep solidphase gene assembly, as well as to assess the performance of different enzymes routinely used for the synthesis and manipulation of DNA (Ref. 31). Moreover, in such assays, the DNA synthesized on the sensor surface can be eluted from the surface by the use of alkali or released by the use of restriction endonucleases, collected and further used for PCR amplification or other applications. Such manipulations are impossible with conventional techniques.

\section{Summary}

In conclusion, biosensors are versatile tools with a range of applications. With a thorough knowledge of possible artefacts and limitations, it possible to perform assays that were heretofore not practicable in immunology.

Sulabha Pathak and Hub Savelkoul (smelkoul@immu.fgg.eur.nl) are at the Dept of Immunology, Erasmus University, PO Box 1738, 3000 DR Rotterdan. The Netherlands.

\section{References}

1 Malmqvist, M. (1993) Curr. Opin. lmmunol. 5, 282-286

2 Steward. M.W. and Lew. A.M. (1985)

J. Imminul. Methods 78, 173-190

3 Butler, J.E., Ni, L., Nessler, R, ef al. (1992)

1. humunol. Methods $150,77-90$

4 Nygren, H., Werthen, M. and Stenberg, $M$. (1987) J. Immunol. Mithods 101, 63-71

5 Shuck, P. (1996) Biophts. I. 70, 1230-1249
6 Karlsion, R. and Falt, A. $\{140,7\}$ immentid Metluts: 2(4), 12I-133

7 Gill, A., Leatherbarrow, R.J., Heare, U Pollard-Knight, D.V. Lowe, I'A and Fortume

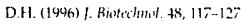

8 VanCout, T.C., L.emis, L.D. Rtutield, R.R. and

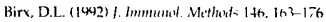
9 Pathak, S.S., Tibbe, Ci.M. M. and Sevelkent

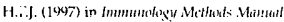
(Lefkovits, 1.. ed.). pp. 1078 1(k)4, Academie Press 10 Malmborg, A-C., Michaclsson, A., Ohlin, V., Jansson, B. and Borrebaek, C.A.K. (1442) Sumid/. Immunol. 35, (3+t?-65)

11 O'Sh.annessy, D. ., Brightam-Burke, .I. Soneson, K.K. and tlensley, P. (1993), 4hil. Bithly. 212, $457-468$

12 Morton, T.A., Myszka, D.C. and Chaike'n. 1.M. (1995) Anul. Bixhem. 227, 176-1,55

13 Edwards, P.R., C.ll, A., Pollard-Kuight. D.V. it al. 11495) Arutl. Biochtm. 231, 210-217

14 Mattes, M.J. (1995) Cancir Ris. 55 . $5733 \mathrm{~s}-5735 \mathrm{~s}$

15 Van.der Merwe, E., Brown, M.H., D.wis, S.J. and Barclay, A.N. (1993) EMBC /. 12, 7945-4454 16 Wu, Z., Johnson, K.W., Choi, Y, and Ciardelli, T.L. (1995) /. Bivl. Clitm. 270). 16045-16151

17 Gertler, A., Granchade, J., Strabturger, C.1.

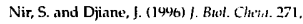
24482-24491

18 Davies, R.J. and Pollard-Knight, D. (1493) Am. Biolech. Lah. July, 1-3

19 Marks, J.D., Griffiths, A.D., Malmquvist, M., Clackson, T., Bye, J.M. and Winter, G. (1442)

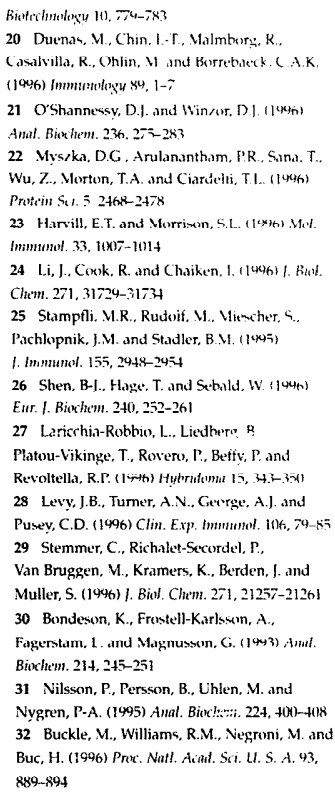

\section{Coming soon in IT}

- The biological relevance of polykaryons in the immune response

- The immunoregulatory effects of natural killer celis: the role of TGF- $\beta$ and implications for autoimmunity

\section{- Feedback regulatory pathways in the thymus}

- $\mathrm{CDA}^{+} \mathrm{T}$-cell subsets and cytokines involoved in peripheral tolerance

- Molecular origin and evolution of $V_{H}$ domains of jawed vertebrates

- Local instability predictive of T-cell epitopes

- The enhancer shift: a model to explain the developmental control of $\operatorname{lgH}$ expression in B-lineage cells

- The molecular basis of immunosenescence

Drn't miss these and many other articles of interest: subscribe to Immenology Today. 